\title{
Clinical Features and Prognostic Impact of Coexpression Modules Constructed by WGCNA for Diffuse Large B-Cell Lymphoma
}

\author{
Jianjun Xiao, ${ }^{1}$ Xuemei Wang, ${ }^{2}$ and Haitao Bai $\mathbb{D}^{3}$ \\ ${ }^{1}$ Department of Oncology, Shaoxing Second Hospital, No. 123 Yan'an Road, Yuecheng District, Shaoxing, \\ 312000 Zhejiang Province, China \\ ${ }^{2}$ Department of Cardiology, Obstetrics and Gynecology Hospital of Fudan University, No. 419, Fangxie Road, Huangpu District, \\ Shanghai 200011, China \\ ${ }^{3}$ Department of Haematology, Shanghai General Hospital of Nanjing Medical University, No. 100 Haining Road, Hongkou District, \\ Shanghai 200080, China
}

Correspondence should be addressed to Haitao Bai; baihaitao123456@sohu.com

Received 1 May 2019; Revised 6 February 2020; Accepted 4 May 2020; Published 8 June 2020

Academic Editor: Gerald J. Wyckoff

Copyright (C) 2020 Jianjun Xiao et al. This is an open access article distributed under the Creative Commons Attribution License, which permits unrestricted use, distribution, and reproduction in any medium, provided the original work is properly cited.

\begin{abstract}
Objective. Diffuse large B-cell lymphoma (DLBCL) is a highly aggressive malignant tumor, accounting for 30-40\% of nonHodgkin's lymphoma. Our aim was to construct novel prognostic models of candidate genes based on clinical features. Methods. RNA-seq and clinical data of DLBCL were retrieved from TCGA database. Coexpression modules were constructed by WGCNA. Then, we investigated the interactions between modules and clinical features. By overall survival analysis, prognostic candidate genes from modules of interest were identified. A coexpression network of prognostic candidate genes was then constructed through WGCNA. GEPIA was used to analyze the expression of a candidate gene between DLBCL and normal samples. Results. 19 coexpression modules were constructed by 12813 genes from 52 DLBCL samples. The number of genes in modules ranged from 34 to 5457 . We found that the purple module was significantly related with histological type ( $p$ value $=$ 1e-04). Overall survival analysis revealed that MAFA-AS1, hsa-mir-338, and hsa-mir-891a were related with prognosis of DLBCL ( $p$ value $=0.027,0.039$, and 0.022 , respectively). A coexpression network was constructed for the three prognostic genes. MAFA-AS1 was interacted with 36 genes, hsa-mir-891a was interacted with 11 genes, while no gene showed interaction with hsa-mir-338. Using GEPIA, we found that MAFA-AS1 showed low expression in DLBCL samples $(p<0.01)$. Conclusion. We constructed a coexpression module related with histological type and identified three candidate genes (MAFA-AS1, hsa-mir338, and hsa-mir-891a) that possessed potential value as prognostic biomarkers and therapeutic targets of DLBCL.
\end{abstract}

\section{Introduction}

DLBCL is a highly aggressive malignant tumor originating from mature B-cells, accounting for 30-40\% of nonHodgkin's lymphoma $[1,2]$. Patients with DLBCL usually have a poor prognosis due to ineffective primary and second-line therapy or recurrence after stem cell transplantation [3]. Therefore, easily applicable prognostic parameters are necessary for clinicians, especially since new molecular markers have not yet entered clinical routines [4]. The International Prognostic Index (IPI) is the most common tool for risk stratification in DLBCL. However, due to improved treatment options, pathobiology, and life expectancy of patients with DLBCL, IPI has been challenged [5]. Therefore, it is necessary to propose novel prognostic models based on clinical features and prognostic biomarkers.

As a molecular heterogeneous disease composed of different histopathologic and genetic subtypes, genetics of DLBCL has clinical implications for patient risk prediction and treatment [6]. Molecular traits are increasingly being used to guide DLBCL drug development, predict patients' clinical outcomes, and make treatment plans [7]. Therefore, the collection and evaluation of molecular features in clinical samples are critical to improve the prognosis of patients 
with DLBCL. Genomic studies have revealed a large number of mutant genes in DLBCL; their clinical significance remains unclear.

As a powerful method for transcriptomics analysis, RNA sequencing (RNA-seq) has been widely used to explore gene function and biological patterns, as well as to find candidate drug targets and to identify biomarkers for predicting disease risk and prognosis $[8,9]$. The Cancer Genome Atlas (TCGA) has produced RNA-seq data, which provides an unprecedented opportunity for cancer biology. Weighted gene coexpression network analysis (WGCNA) is a systematic biological method, which is widely used to generate gene coexpression networks [10]. Instead of linking thousands of genes to physiological characteristics, it focuses more on the relationship between several modules and features [11]. It provides a specific measure for clinical prediction of DLBCL diagnosis and developing new treatment strategies [12]. Therefore, WGCNA can explore hidden biological patterns. The method has been used to analyze many kinds of diseases, such as breast cancer, uveal melanoma, gastric cancer, and colon cancer [13-16]. However, there is no study on clinical modules of DLBCL using the WGCNA method.

In the present study, coexpression modules were constructed by WGCNA. After investigating the correlations between modules and clinical features, a module of interest was identified for functional enrichment analysis. We found three candidate genes (MAFA-AS1, hsa-mir-891a, and hsamir-338) that were related with prognosis of DLBCL by overall survival analysis. And MAFA-AS1 had low expression in DLBCL by GEPIA.

\section{Materials and Methods}

2.1. Data Processing. RNA-seq and clinical data of lymphoid neoplasm diffuse large B-cell lymphoma patients were downloaded from TCGA data repository (https://cancergenome .nih.gov/). The gene expression level was normalized as fragments per kilobase of transcript per million mapped reads (FPKM) using Robust Multiarray Average (RMA) algorithm. The miRNA expression level was measured as RPM. Clinical information included clinical TNM stage, histologic grade, age, gender, and survival information. As genes with little variation in expression usually represent noise, the most variant genes were filtered. Gene variables were measured by median absolute deviation (MAD).

2.2. Construction of Gene Coexpression Network. Gene coexpression network was constructed using WGCNA package in $\mathrm{R}$ [17]. Power values were screened out by the WGCNA algorithm. Firstly, gene expression similarity matrix $\mathrm{S}=\left(s_{i j}\right)$ was constructed via calculating the absolute value of Pearson correlation coefficient between two genes. The formula was listed as below:

$$
s_{i j}=\left|\frac{1+\operatorname{cor}\left(\mathrm{x}_{\mathrm{i}}, \mathrm{x}_{\mathrm{j}}\right)}{2}\right| \text {, }
$$

TABLE 1: Clinical features of DLBCL patients.

\begin{tabular}{lccc}
\hline Clinical features & Total $(n=47)$ & Alive $(n=38)$ & Dead $(n=9)$ \\
\hline $\begin{array}{l}\text { Age, mean } \\
\text { (range) }\end{array}$ & $56.3(23-82)$ & $56.8(23-82)$ & $54.1(27-73)$ \\
Gender & & & \\
$\quad$ Male & 22 & 17 & 5 \\
$\quad$ Female & 25 & 21 & 4 \\
Stage & & & \\
I & 7 & 6 & 1 \\
II & 17 & 15 & 2 \\
III & 5 & 4 & 1 \\
IV & 12 & 9 & 3 \\
$\quad-$ & 6 & 4 & 2 \\
Race & & 14 & 4 \\
$\quad$ Asian & 18 & 24 & 4 \\
White & 28 & 0 & 1 \\
Black or African & 1 & & \\
American & & & \\
\hline
\end{tabular}

where $\mathrm{x}_{\mathrm{i}}$ and $\mathrm{x}_{\mathrm{j}}$ were vectors of expression value for gene $i$ and $j$ and $s_{i j}$ represented the Pearson correlation coefficient of gene $i$ and gene $j$.

Next, gene expression similarity matrix was transformed into adjacency matrix $\mathrm{a}_{\mathrm{ij}}$. The formula was $\mathrm{a}_{\mathrm{ij}}=\left|s_{i j}\right|^{\beta}$. To further identify functional modules in the coexpression network, the adjacent matrix was transformed into a topological overlap matrix (TOM), and the corresponding dissimilarity (1-TOM) was calculated.

$$
\operatorname{tom}_{i j}=\frac{\sum_{\mu \neq i, j} a_{i \mu} a_{\mu j}+a_{i j}}{\min \left(\sum_{\mu} a_{i \mu}, \sum_{\mu} a_{\mu j}\right)+1-a_{i j}}
$$

where $\alpha$ was the weighted adjacency matrix given by $\mathrm{a}_{\mathrm{ij}}=$ $\left|s_{i j}\right|^{\beta}$ and $\beta=5$ was the soft threshold power.

$$
D=\left(\begin{array}{c}
d_{1} \\
d_{2} \\
\vdots \\
d_{n}
\end{array}\right),
$$

where $D$ expressed the degree of dissimilarity gene expression in different samples.

Scale independence and average connectivity analysis of modules with different power values was performed by gradient test (power value ranging from 1 to 20). Appropriate power value was determined when the scale independence value was equal to 0.9 . The WGCNA algorithm was then used to construct the coexpression network and extract the gene information in the most relevant module. The candidate network was selected according to the coexpression weight $>2.5$. 


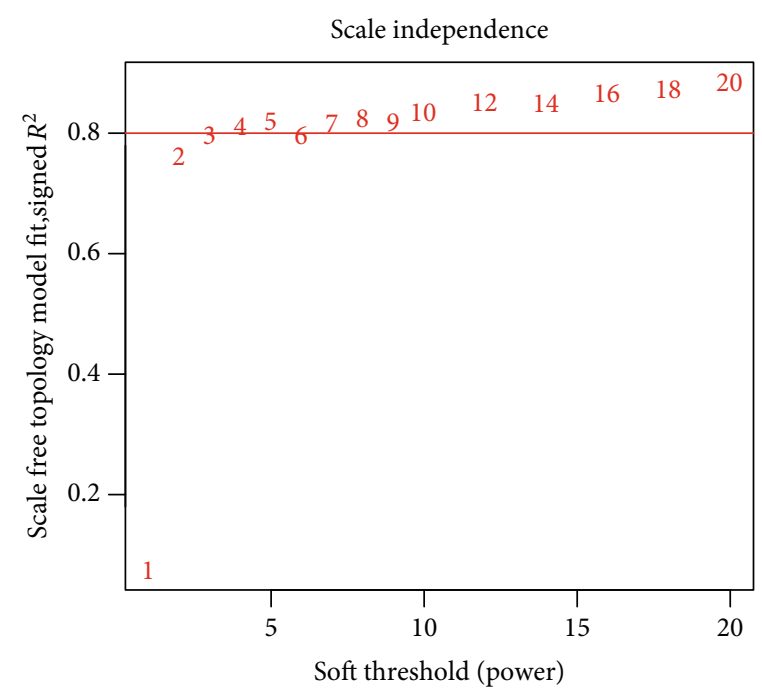

(a)

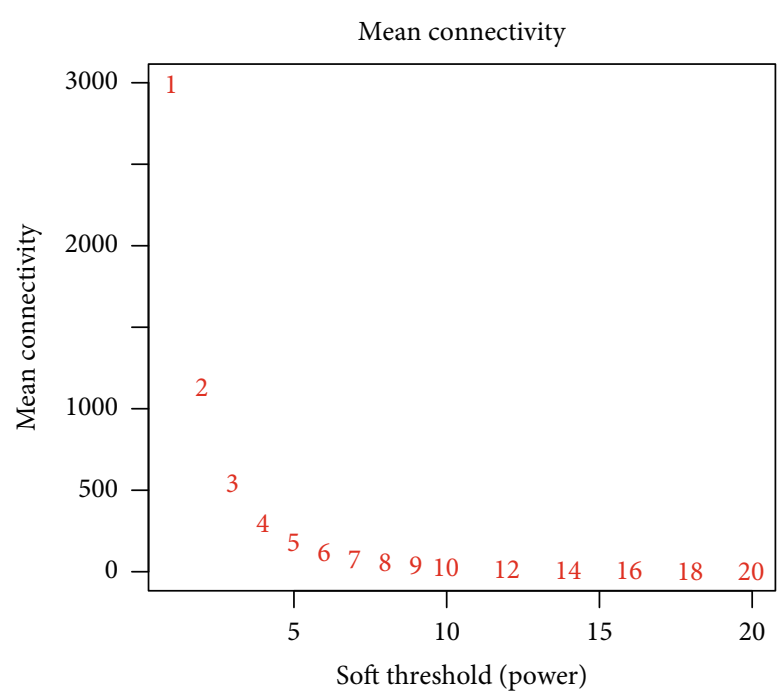

(b)

FIGURE 1: Analysis of network topology for various soft-thresholding powers: (a) the scale-free fit index as a function of the soft-thresholding power. The $x$-axis represents the soft-thresholding power and the $y$-axis represents the scale-free fit index; (b) the mean connectivity as a function of the soft-thresholding power. The $x$-axis stands for the soft-thresholding power and the $y$-axis stands for the mean connectivity.

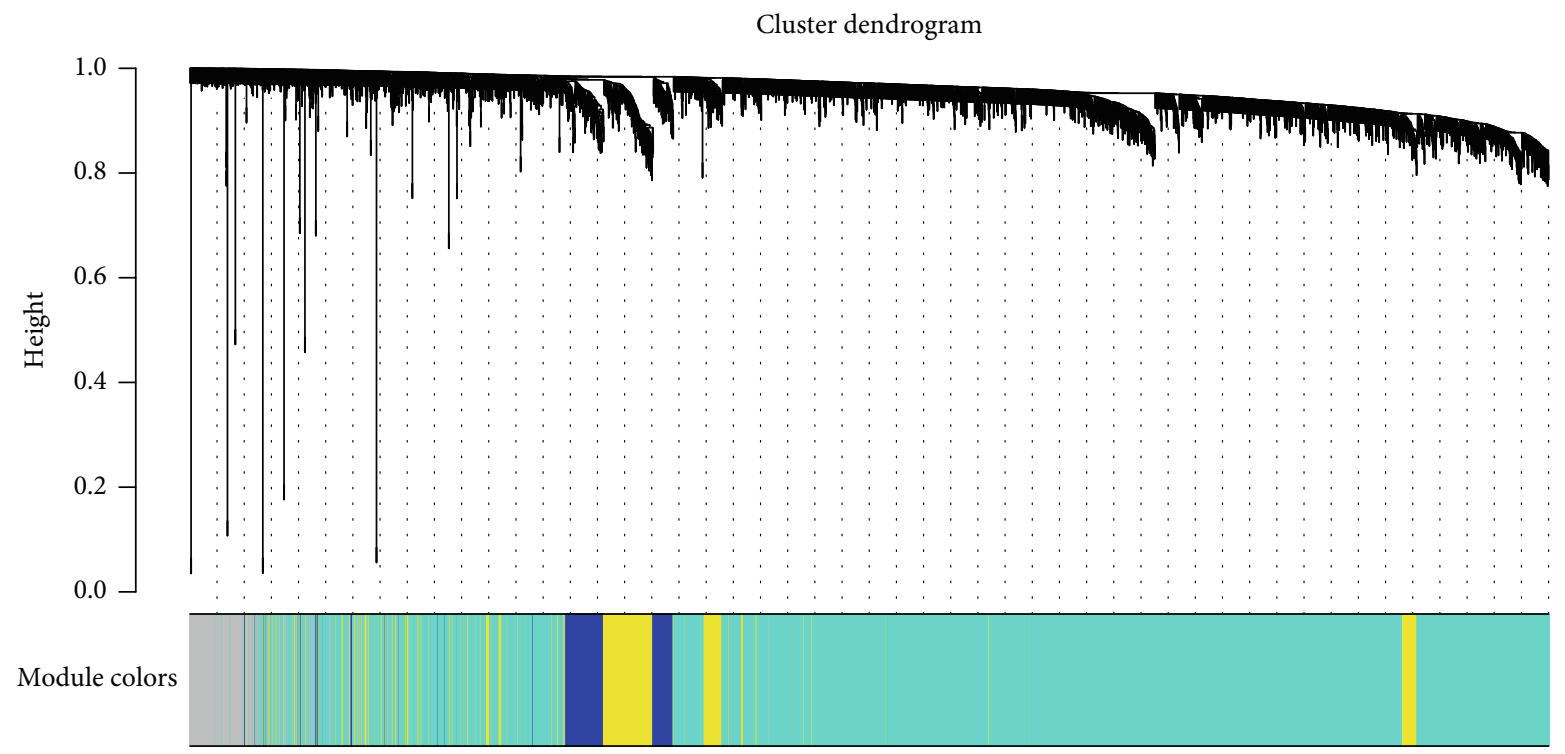

FIgURE 2: Cluster dendrogram obtained by average linkage hierarchical clustering. The color below the dendrogram demonstrates the module assignment determined by the dynamic tree cut. 19 coexpression modules were depicted in different colors. The number of genes in coexpression modules ranged from 34 to 5457 .

2.3. Correlations between MEs and External Clinical Data. The correlations between modules and clinical features were analyzed using WGCNA. Module eigengene (ME) can summarize the gene expression profiles, and the formula was as follows:

$$
\operatorname{ME}_{i}^{q}=\operatorname{princomp}\left(x_{i j}^{(q)}\right)
$$

where $q$ represents the $q$ th module.
We calculated the correlations between MEs and external clinical data as the module membership (MM). $p<0.05$ was statistically significant. The genes in the most relevant module were chosen as candidate genes, as follows:

$$
\mathrm{MM}_{i}^{q}=\operatorname{cor}\left(x_{i}, \mathrm{ME}_{i}^{q}\right)
$$

where $\mathrm{ME}_{i}^{q}$ means the identification of the $i$ th gene in the $q$ th module. 


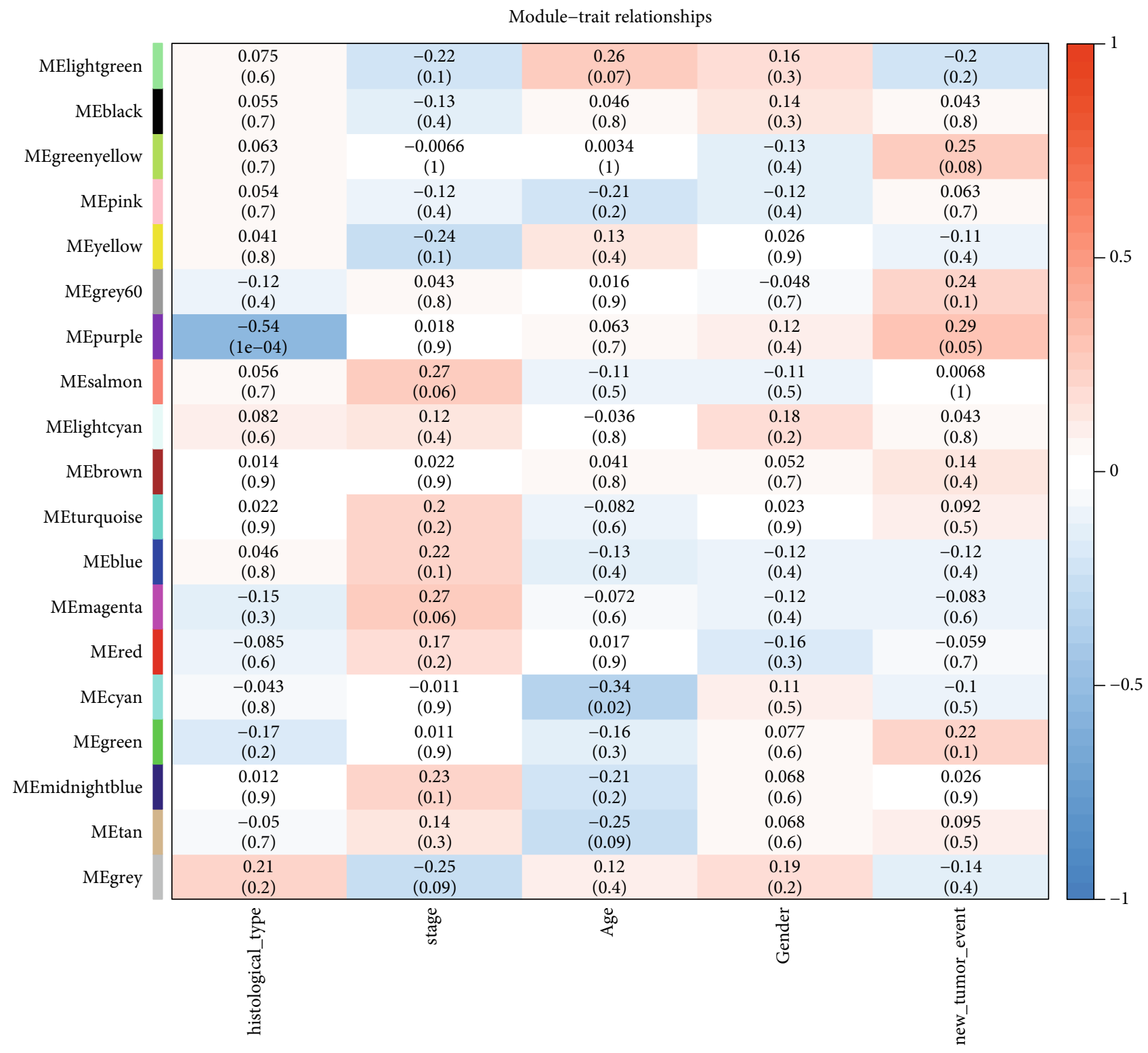

FIGURE 3: Module-trait relationships. Each row represents a module eigengene, and column represents a trait. Each cell contains the corresponding correlation and $p$ value. The table is color-coded by correlation based on the color legend.

We calculated the correlations between MEs and external clinical data. A $p$ value $<0.05$ was statistically significant. The genes in the most relevant module were chosen as candidate genes.

2.4. Functional Enrichment Analysis. To explore the potential biological themes and pathways of genes from modules of interest, the clusterProfiler package in $\mathrm{R}$ was used to annotate and visualize Gene Ontology (GO) terms (including biological processes, molecular functions, and cellular components) and Kyoto Encyclopedia of Genes and Genomes (KEGG) pathways [18]. A $p$ value $<0.05$ was considered significant pathways.

2.5. Prognostic Analysis of Candidate Module Genes. Then, we further assessed the prognostic value of candidate genes by overall survival analysis. Log-rank tests were used to select prognosis-related genes from candidate genes. Survival package was used to carry out log-rank tests and survminer package was used to plot Kaplan-Meier survival curves.

2.6. Candidate Gene Coexpression Network Construction. WGCNA can evaluate coexpression information of genes. Through survival analysis, we obtained the core genes related with prognosis. Moreover, we constructed a coexpression network of prognosis-related genes through WGCNA.

2.7. Candidate Gene Risk Assessment. The online database Gene Expression Profiling Interactive Analysis (GEPIA) (http://gepia.cancer-pku.cn/index.html) was used to analyze the gene expression between cancer and normal samples [19]. As an interactive web, GEPIA can analyze the RNA- 
TABLE 2: GO enrichment analysis results of genes in the purple module.

\begin{tabular}{|c|c|c|c|c|}
\hline Term ID & Ontology & Description & p.adjust & Count \\
\hline GO:0048167 & $\mathrm{BP}$ & Regulation of synaptic plasticity & 0.00573 & 7 \\
\hline GO:0021782 & $\mathrm{BP}$ & Glial cell development & 0.01269 & 5 \\
\hline GO:0022010 & $\mathrm{BP}$ & Central nervous system myelination & 0.01269 & 3 \\
\hline GO:0032291 & $\mathrm{BP}$ & Axon ensheathment in central nervous system & 0.01269 & 3 \\
\hline GO:0042063 & $\mathrm{BP}$ & Gliogenesis & 0.01776 & 7 \\
\hline GO:0010975 & $\mathrm{BP}$ & Regulation of neuron projection development & 0.01776 & 9 \\
\hline GO:0010001 & $\mathrm{BP}$ & Glial cell differentiation & 0.01902 & 6 \\
\hline GO:0085029 & $\mathrm{BP}$ & Extracellular matrix assembly & 0.02101 & 3 \\
\hline GO:0048708 & $\mathrm{BP}$ & Astrocyte differentiation & 0.02214 & 4 \\
\hline GO:0050804 & BP & Modulation of chemical synaptic transmission & 0.02299 & 8 \\
\hline GO:0099177 & $\mathrm{BP}$ & Regulation of transsynaptic signaling & 0.02299 & 8 \\
\hline GO:0060291 & $\mathrm{BP}$ & Long-term synaptic potentiation & 0.02542 & 4 \\
\hline GO:0014002 & $\mathrm{BP}$ & Astrocyte development & 0.02736 & 3 \\
\hline GO:0110110 & $\mathrm{BP}$ & Positive regulation of animal organ morphogenesis & 0.02776 & 4 \\
\hline GO:0001505 & $\mathrm{BP}$ & Regulation of neurotransmitter levels & 0.03131 & 7 \\
\hline GO:0014003 & $\mathrm{BP}$ & Oligodendrocyte development & 0.03886 & 3 \\
\hline GO:0001657 & $\mathrm{BP}$ & Ureteric bud development & 0.03886 & 4 \\
\hline GO:0072163 & $\mathrm{BP}$ & Mesonephric epithelium development & 0.03886 & 4 \\
\hline GO:0072164 & $\mathrm{BP}$ & Mesonephric tubule development & 0.03886 & 4 \\
\hline GO:0001504 & $\mathrm{BP}$ & Neurotransmitter uptake & 0.04008 & 3 \\
\hline GO:0001823 & $\mathrm{BP}$ & Mesonephros development & 0.04097 & 4 \\
\hline GO:0050768 & $\mathrm{BP}$ & Negative regulation of neurogenesis & 0.04282 & 6 \\
\hline GO:0006953 & $\mathrm{BP}$ & Acute-phase response & 0.04605 & 3 \\
\hline GO:0016264 & $\mathrm{BP}$ & Gap junction assembly & 0.04605 & 2 \\
\hline GO:0071281 & $\mathrm{BP}$ & Cellular response to iron ion & 0.04605 & 2 \\
\hline GO:0014013 & $\mathrm{BP}$ & Regulation of gliogenesis & 0.04823 & 4 \\
\hline GO:0051961 & $\mathrm{BP}$ & Negative regulation of nervous system development & 0.04823 & 6 \\
\hline GO:0042552 & $\mathrm{BP}$ & Myelination & 0.04823 & 4 \\
\hline GO:0007272 & $\mathrm{BP}$ & Ensheathment of neurons & 0.04823 & 4 \\
\hline GO:0008366 & $\mathrm{BP}$ & Axon ensheathment & 0.04823 & 4 \\
\hline GO:0043209 & $\mathrm{CC}$ & Myelin sheath & 0.00032 & 7 \\
\hline GO:0014069 & $\mathrm{CC}$ & Postsynaptic density & 0.00777 & 7 \\
\hline GO:0032279 & $\mathrm{CC}$ & Asymmetric synapse & 0.00777 & 7 \\
\hline GO:0099572 & $\mathrm{CC}$ & Postsynaptic specialization & 0.00777 & 7 \\
\hline GO:0098984 & $\mathrm{CC}$ & Neuron to neuron synapse & 0.00777 & 7 \\
\hline GO:0097449 & $\mathrm{CC}$ & Astrocyte projection & 0.02459 & 2 \\
\hline GO:0043083 & $\mathrm{CC}$ & Synaptic cleft & 0.02892 & 2 \\
\hline GO:0098793 & $\mathrm{CC}$ & Presynapse & 0.03602 & 7 \\
\hline GO:0031012 & $\mathrm{CC}$ & Extracellular matrix & 0.03602 & 7 \\
\hline GO:0033267 & $\mathrm{CC}$ & Axon part & 0.03871 & 6 \\
\hline GO:0030426 & $\mathrm{CC}$ & Growth cone & 0.04208 & 4 \\
\hline GO:0030427 & $\mathrm{CC}$ & Site of polarized growth & 0.04208 & 4 \\
\hline GO:0097386 & $\mathrm{CC}$ & Glial cell projection & 0.04208 & 2 \\
\hline GO:0150034 & $\mathrm{CC}$ & Distal axon & 0.04208 & 5 \\
\hline GO:0034364 & $\mathrm{CC}$ & High-density lipoprotein particle & 0.04689 & 2 \\
\hline GO:0048306 & MF & Calcium-dependent protein binding & 0.01134 & 4 \\
\hline GO:0005539 & MF & Glycosaminoglycan binding & 0.01134 & 6 \\
\hline GO:0048018 & MF & Receptor ligand activity & 0.01134 & 8 \\
\hline
\end{tabular}


TABLE 2: Continued.

\begin{tabular}{lccc}
\hline Term ID & Ontology & Description & p.adjust \\
\hline GO:0008201 & MF & Heparin binding & 0.01134 \\
GO:0004857 & MF & Enzyme inhibitor activity & 0.01134 \\
GO:0005381 & MF & Iron ion transmembrane transporter activity & 7 \\
GO:1901681 & MF & Sulfur compound binding & 0.02116 \\
\hline
\end{tabular}

BP: biological processes; CC: cellular components; MF: molecular functions.

seq expression including TCGA datasets and GenotypeTissue Expression (GTEx) datasets [20]. Using GEPIA, we analyzed candidate genes related with prognosis between cancer and normal samples from TCGA.

2.8. Reverse-Transcription Quantitative PCR (RT-qPCR). Total RNA was extracted from plasma of 11 patients with DLBCL and 11 healthy participates using Trizol. To examine hsa-miR-338-3p, hsa-mir-338-5p, and hsa-miR-891a-5p expression, cDNA was synthesized with the miScript Reverse Transcription Kit (Qiagen, Hilden, Germany). RT-qPCR was performed using the miScript SYBR Green PCR Kit (Qiagen). $\beta$-Actin served as an internal control. The relative expression levels of miRNAs were calculated with the $2^{-\Delta \Delta \mathrm{Ct}}$ method. The specific primers for hsa-miR-338-3p, hsa-mir338-5p, and hsa-miR-891a-5p were as follows: hsa-miR-3383p, $5^{\prime}$-AACCGGTCCAGCATCAGTGATT-3' (forward), $5^{\prime}$ GTGCAGGGTCCGAGGT-3' (reverse); hsa-mir-338-5p, $5^{\prime}$ CAATATCCTGGTGCTGAGTG-3' (forward), $5^{\prime}$-GTGCA GGGTCCGAGGT-3' (reverse); and hsa-miR-891a-5p, $5^{\prime}$-G TGCTCGCTTCGGCAGCACATA-3' (forward), 5' -GTGCA GGGTCCGAGGT-3' (reverse).

\section{Results}

3.1. Gene Coexpression Network of DLBCL. Clinical and level3 RNA-seq data of 51 DLBCL samples were retrieved from TCGA. The clinical features of the DLBCL samples are listed in Table 1. After module detection, 12813 most variant genes were selected for further analysis according to MAD value when the value of soft thresholding power $\beta$ was 12 , and the connectivity between genes met a scale-free network distribution (Figure 1). 19 modules were identified by hierarchical clustering and the dynamic branch cutting (Figure 2). Each module was assigned a unique color as an identifier. The number of genes in modules ranged from 34 to 5457 . The grey module represented a gene set that was not assigned to any of the modules.

3.2. Identification of the Purple Module as the Module Most Relevant to Clinical Traits. To explore the molecular mechanisms behind the trait, we identified genes associated with a certain clinical trait. In the present study, the clinical parameters of DLBCL patients, including age, clinical stage, gender, recrudescence stage, and histological type were involved in the module-trait relationship analysis. As shown in Figure 3, the purple module was closely related with histological type $(p$ value $=1 e-04)$ and the cyan module was associated with age $(p$ value $=0.02)$. Therefore, the purple module was selected as module of interest for subsequent analysis.

3.3. Functional Enrichment Analysis of Genes in the Purple Module. To explore potential pathways of the purple module, GO and KEGG enrichment analyses were performed on the genes from the purple module. $52 \mathrm{GO}$-enriched terms were shown Table 2. The top ten GO terms included regulation of neuron projection development, gliogenesis, regulation of synaptic plasticity, myelin sheath, glial cell differentiation, glial cell development, calcium-dependent protein binding, extracellular matrix assembly, axon ensheathment in central nervous system, and central nervous system myelination (Figure 4(a)). In the KEGG analysis, 10 pathways were enriched by genes in the purple module, including synaptic vesicle cycle, Ras signaling pathway, GABAergic synapse, morphine addiction, cell adhesion molecules (CAMs), PI3K-Akt signaling pathway, retrograde endocannabinoid signaling, gastric cancer, histidine metabolism, and reninangiotensin system (Table 3, Figure 4(b)).

3.4. MAFA-AS1, hsa-mir-338, and hsa-Mir-891a as Candidate Genes Related with Prognosis of DLBCL. The purple module was closely related with histological type. Therefore, genes in the module were of great significance to evaluate the potential value. Among the 100 genes, 3 genes (MAFA-AS1, hsa-mir-338, and hsa-mir-891a) were associated with prognosis of DLBCL (Table 4). As shown in Figure 5, highly expressed MAFA-AS1 and hsa-mir-338 had shorter survival time than their low expression $(p$ value $=0.027$ and 0.039 , respectively). And low expression of hsa-mir-891a had poorer clinical outcomes than its high expression $(p$ value $=$ 0.022).

3.5. Candidate Gene Coexpression Network Construction. We constructed a coexpression network for genes with prognostic significance by WGCNA. As shown in Figure 6, MAFAAS1 was interacted with 36 genes, hsa-mir-891a was interacted with 11 genes, while no gene showed interaction with hsa-mir-338.

3.6. Candidate Gene Risk Assessment. Using GEPIA datasets, we found that MAFA-AS1 showed low expression in DLBCL samples $(p<0.01$, Figure 7).

3.7. Validation of Candidate Prognostic miRNAs. Consistent with our bioinformatics results, RT-qPCR results showed that hsa-miR-338-3p $\quad(p<0.01)$ and hsa-miR-891a-5p $(p<0.001)$ were upregulated in DLBCL (Figure 8$)$. Furthermore, we further validated the candidate prognostic miRNAs 


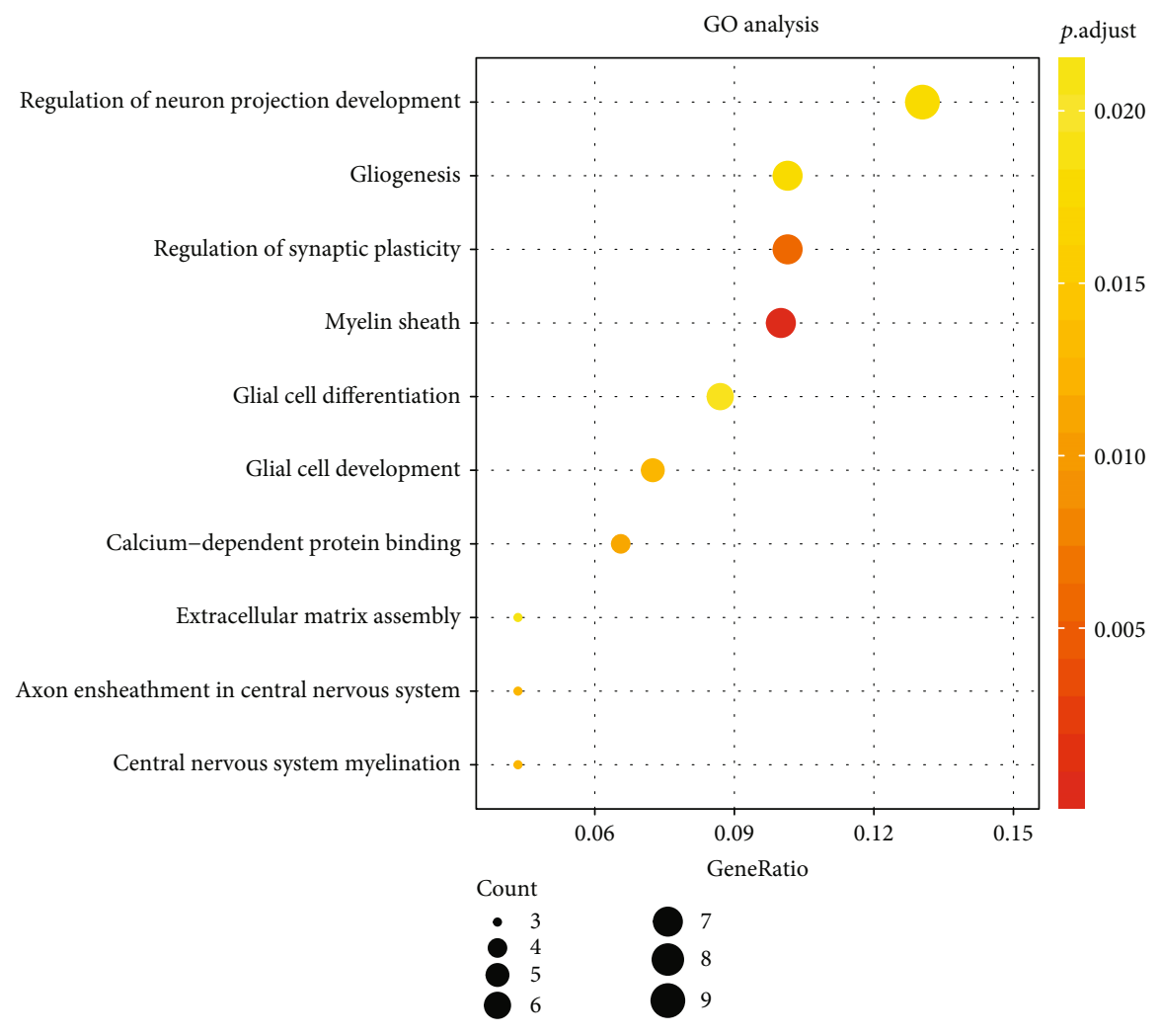

(a)

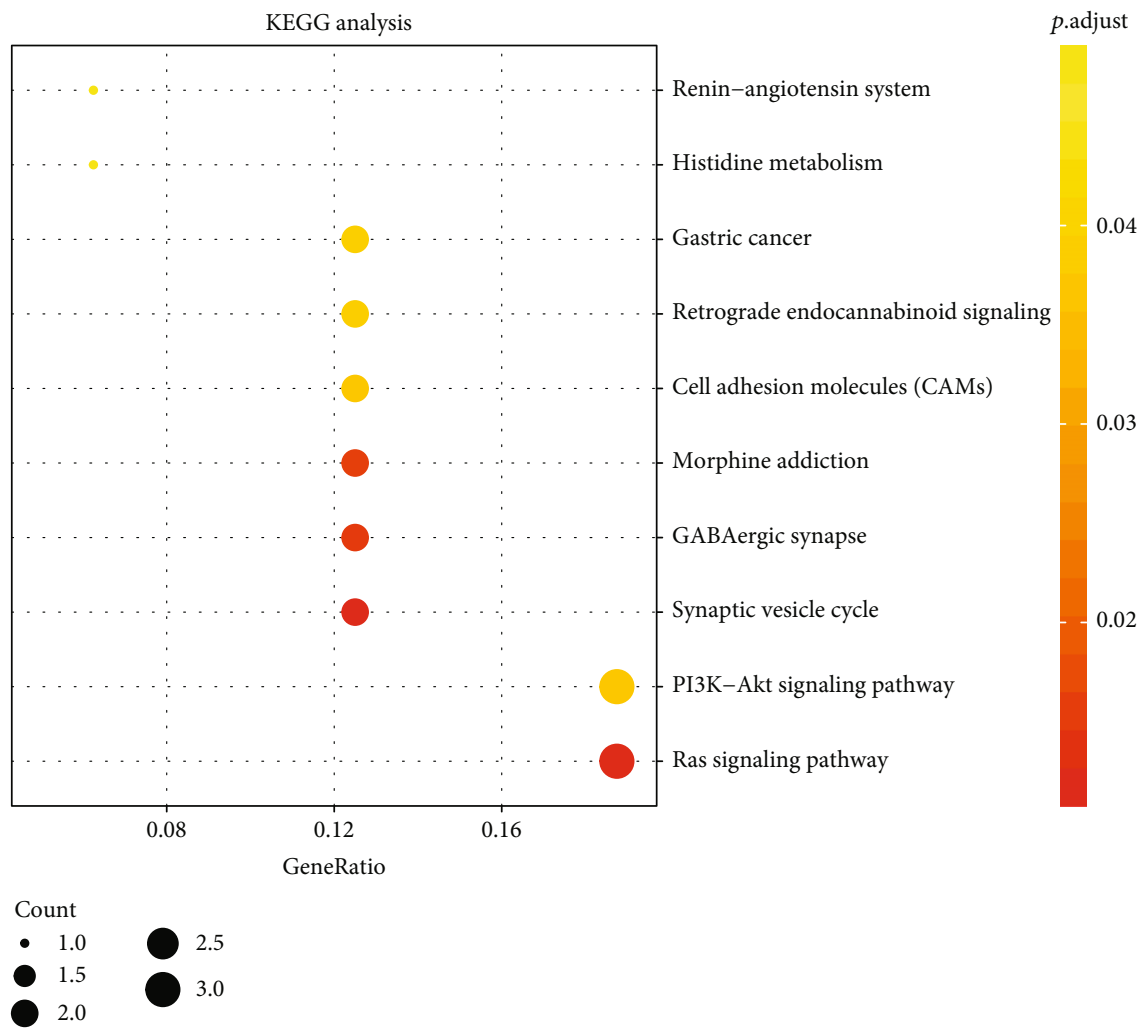

(b)

FIGURE 4: Functional enrichment analysis results of genes in the purple module: (a) the top ten GO terms of genes in the purple module; (b) KEGG pathways of genes in the purple module. 
TABLE 3: KEGG pathway enrichment analysis results of genes in the purple module.

\begin{tabular}{lccc}
\hline ID & Description & p.adjust & Count \\
\hline hsa04721 & Synaptic vesicle cycle & 0.01171 & 2 \\
hsa04014 & Ras signaling pathway & 0.01222 & 3 \\
hsa04727 & GABAergic synapse & 0.01507 & 2 \\
hsa05032 & Morphine addiction & 0.01572 & 2 \\
hsa04514 & Cell adhesion molecules (CAMs) & 0.03700 & 2 \\
hsa04151 & PI3K-Akt signaling pathway & 0.03718 & 2 \\
hsa04723 & Retrograde endocannabinoid signaling & 0.03890 & 2 \\
hsa05226 & Gastric cancer & 0.03938 & 1 \\
hsa00340 & Histidine metabolism & 0.04811 & 0.04811 \\
hsa04614 & Renin-angiotensin system & & 1 \\
\hline
\end{tabular}

TABLE 4: Three candidate genes related with prognosis of DLBCL.

\begin{tabular}{lccc}
\hline Gene & Hazard ratio & $95 \%$ CI & $p$ value \\
\hline MAFA-AS1 & 3.794 & $0.96-14.995$ & 0.026604 \\
hsa-mir-338 & 4.185 & $1.127-15.54$ & 0.039354 \\
hsa-mir-891a & 0.205 & $0.055-0.772$ & 0.021503 \\
\hline
\end{tabular}

in independent datasets. Similarly, the results showed that hsa-miR-338 (Figure 9(a)) and hsa-miR-891a (Figure 9(b)) were highly expressed in DLBCL. High expression of hsamiR-338 (Figure 9(c); $p=0.039$ ) and hsa-miR-891a (Figure $9(\mathrm{~d}) ; p=0.048)$ was significantly associated with worse prognosis of DLBCL patients.

\section{Discussion}

As a heterogeneous disease, DLBCL can be classified into activated B-cell, germinal center B-cell, and primary mediastinal B-cell subtypes based on gene expression profiling [21]. Though about $70 \%$ of DLBCL patients have survival time longer than five years when treated with immunochemotherapy involving rituximab plus cyclophosphamide, doxorubicin, vincristine, and prednisolone (R-CHOP) [22], however, the remaining patients are still dying of this malignant tumor. In addition, the short- and long-term toxicity of chemotherapy, including secondary malignancies and leukemia, adversely affects the long-term prognosis of patients. Therefore, it is necessary to study new therapeutic targets in DLBCL.

RNA-seq is a next-generation sequencing technology for genome-wide quantitative gene expression, which has some advantages over microarrays in characterizing transcriptomes $[23,24]$. However, few studies have investigated the network characteristics of coexpression networks based on RNA-seq. In the present study, RNA-seq and clinical data of DLBCL were retrieved from TCGA. 19 coexpression modules were built by the 12813 most variant genes from 51 DLBCL samples using WGCNA. WGCNA has been proved to be an effective method for detecting coexpression modules and hub genes in many aspects [25]. The interaction between genes in different coexpression modules can be found.
Although a large number of biomarkers have been identified and validated, few studies have considered correlations between genes. Genes with similar expression patterns can encode proteins with similar functional properties that can form complexes or can function in similar pathways. Therefore, we made full use of the WGCNA algorithm to detect key genes associated with sample traits in coexpressed gene networks [26]. The purple module was closely related with histology type $(p$ value $=1 e-04)$. And the cyan module was closely related with age. The incidence of DLBCL is positively correlated with age, about two-thirds of DLBCL patients are over 65 years old worldwide [27]. Research has found that age at diagnosis has a relationship with the molecular features of DLBCL [28].

We found that the genes in the purple module were involved in multiple pathways such as Ras signaling pathway, PI3K/AKT signaling pathway, and cell adhesion molecules. The Ras signaling pathway plays an important role in cancer biology, which regulates cell growth and proliferation. Activating mutations in Ras can result in abnormal activation of its downstream target MEK1/2 [29]. MEK has become a potential target for the treatment of DLBCL. The PI3K/AKT signaling pathway is activated in DLBCL, which plays a key role in controlling the proliferation and survival of DLBCL cells [30]. Activation of this pathway in DLBCL can cause gene mutations, loss of PTEN, or constitutive activation of upstream regulatory pathways [31]. Cell adhesion molecules function in complex biological processes like cancer progression, inflammation, angiogenesis, and metastasis [32-34].

miRNAs are involved in several biological processes by regulating gene expression at the posttranscriptional level, such as cell proliferation and apoptosis $[35,36]$. For example, downregulated microRNA-155 promotes cell cycle arrest and apoptosis in DLBCL [37]. And miR-10a suppresses cell proliferation and promotes cell apoptosis via targeting BCL6 in DLBCL [38]. The main reason for the poor results of DLBCL chemotherapy is that DLBCL cells are resistant to chemotherapeutic drugs [39]. It has been confirmed that miRNAs are closely associated with cancer chemosensitivity. To investigate the prognosis value of the genes in the purple module, we made overall survival analysis and results revealed that three miRNAs were closely related with survival. Highly expressed MAFA-AS1 and hsa-mir-338 had shorter survival 

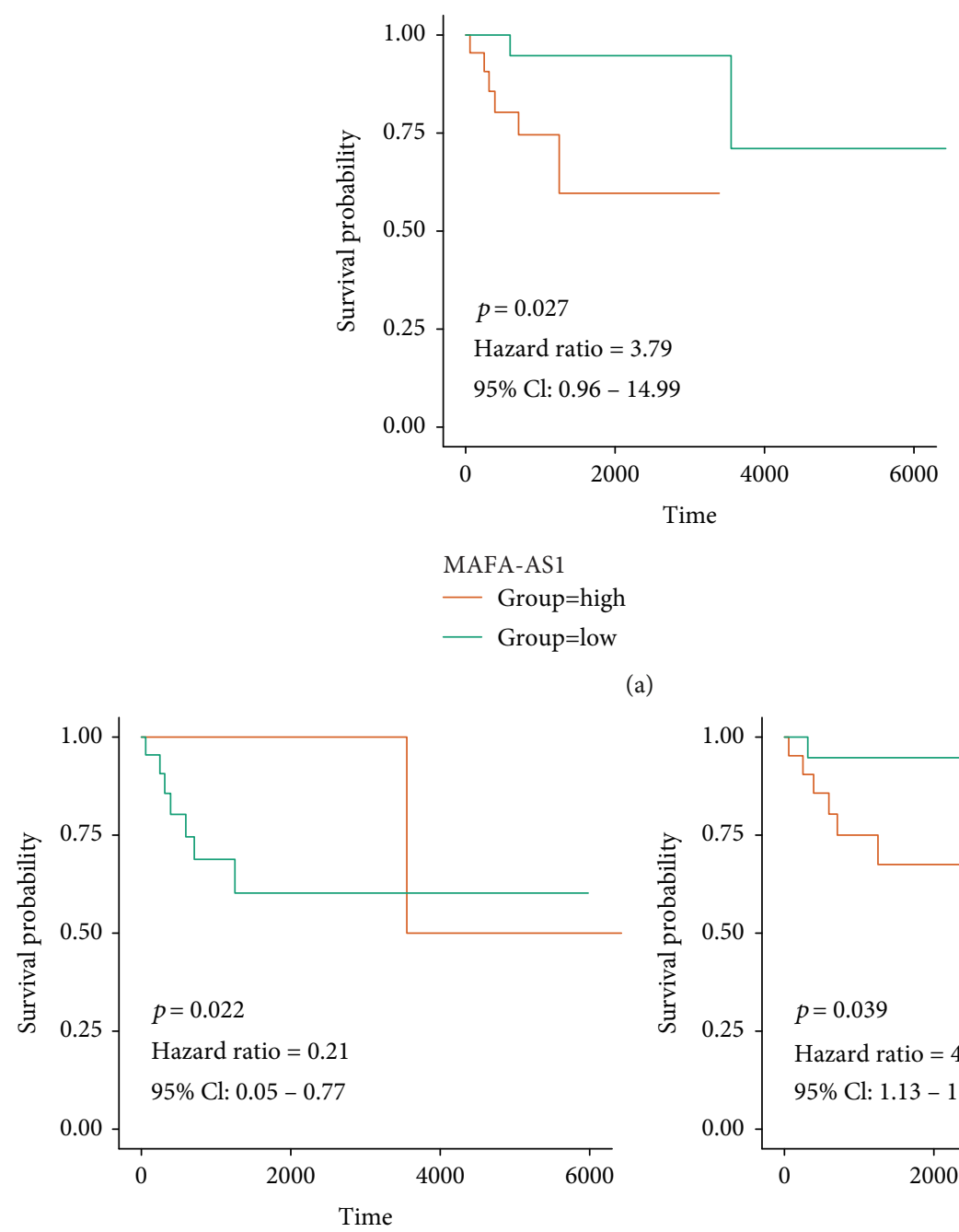

(a)

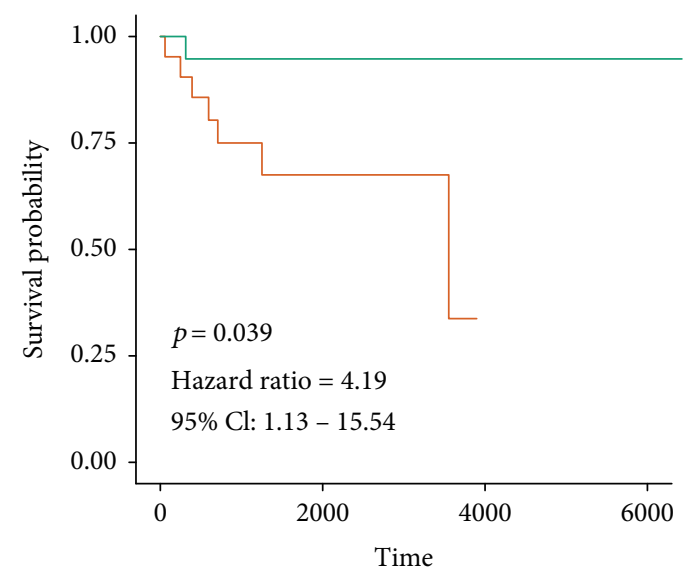

hsa-mir-891a

- Group $=$ high
- Group $=$ low

hsa-mir-338

- Group=high

(b)

(c)

Figure 5: Overall survival analysis of three candidate genes: (a) MAFA-AS1; (b) hsa-mir-891a; (c) hsa-mir-338.

time than their low expression $(p$ value $=0.027$ and 0.039 , respectively). And low expression of hsa-mir-891a had poorer clinical outcomes than its high expression ( $p$ value $=$ 0.022). So far, there is no study concerning MAFA-AS1 and hsa-mir-891a. As for hsa-mir-338, it has been reported that hsa-mir-338-3p inhibits invasion and migration of colorectal cancer cells by suppressing smooth expression [40]. Aberrantly expressed hsa-mir-338-3p increases the risk of esophageal cancer [41]. In addition, hsa-mir-338 can be a prognostic biomarker for oral squamous cell carcinoma [42]. Then, we constructed a coexpression network for the three genes by WGCNA. MAFA-AS1 was interacted with 36 genes, hsa-mir-891a was interacted with 11 genes, while no gene showed interaction with hsa-mir-338. Using GEPIA datasets, we found that MAFA-AS1 showed low expression in DLBCL samples $(p<0.01)$.

Taken together, three candidate genes (MAFA-AS1, hsamir-338, and hsa-mir-891a) from the purple module could be considered prognostic biomarkers for DLBCL. Compared to IPI, the new prognostic model had some advantages. For example, the three candidate genes could be detected using immunohistochemistry, which is convenient to screen patients with DLBCL and predict their prognosis. Moreover, based on clinical features and candidate genes, it can help improve treatment options, pathobiology, and life expectancy of patients with DLBCL. However, several limitations of our study need to be pointed out. The heterogeneity of the treatment protocols is inevitable, which can bias outcomes. Furthermore, the number of samples is limited; therefore, the prognostic role of the three candidate genes requires to be verified in a larger sample of DLBCL.

\section{Conclusion}

In our study, RNA-seq and clinical data of DLBCL were retrieved from TCGA. 19 coexpression modules were built 


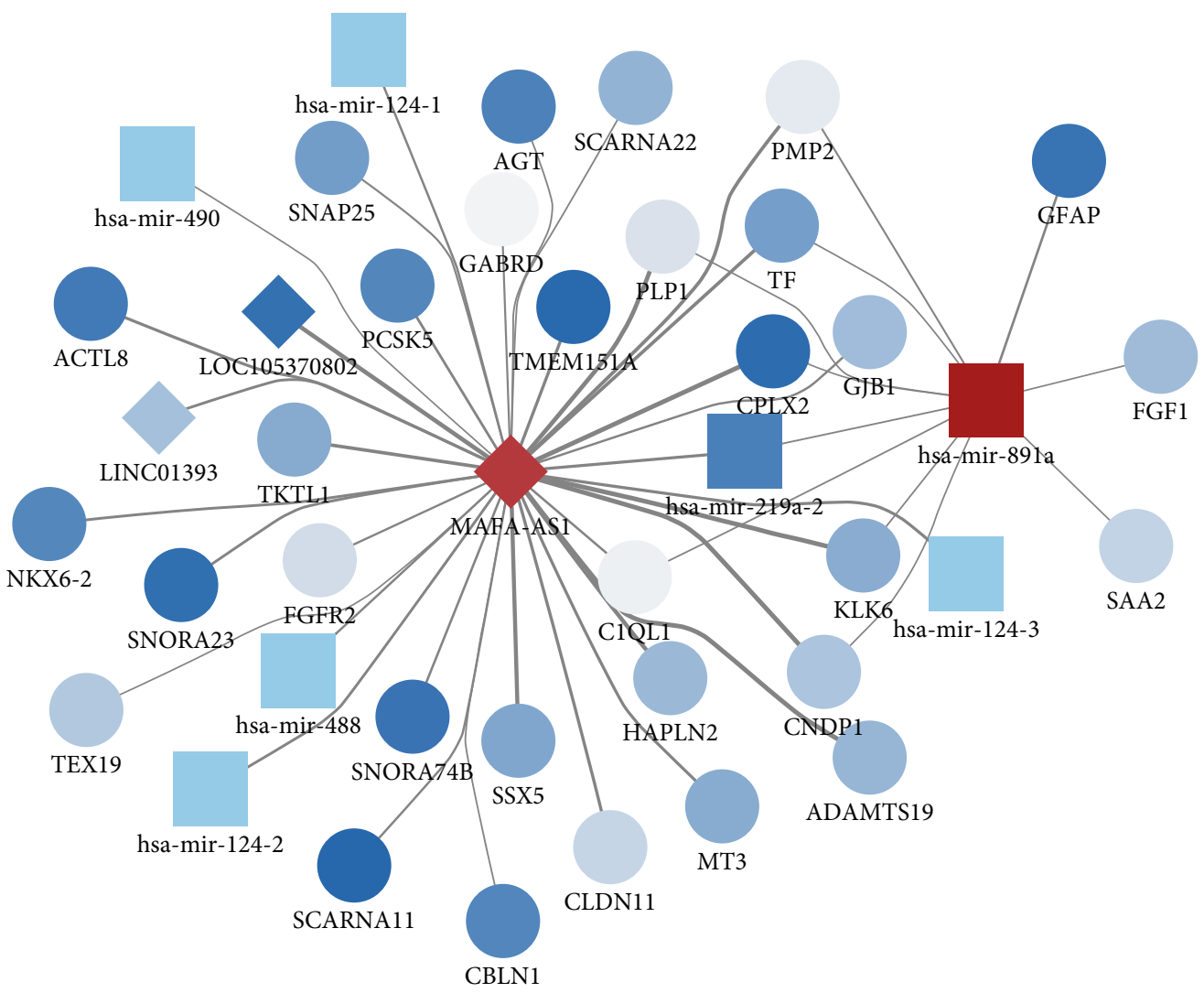

FIGURE 6: Candidate gene coexpression network construction. Round stands for mRNAs and rhombus represents miRNAs.

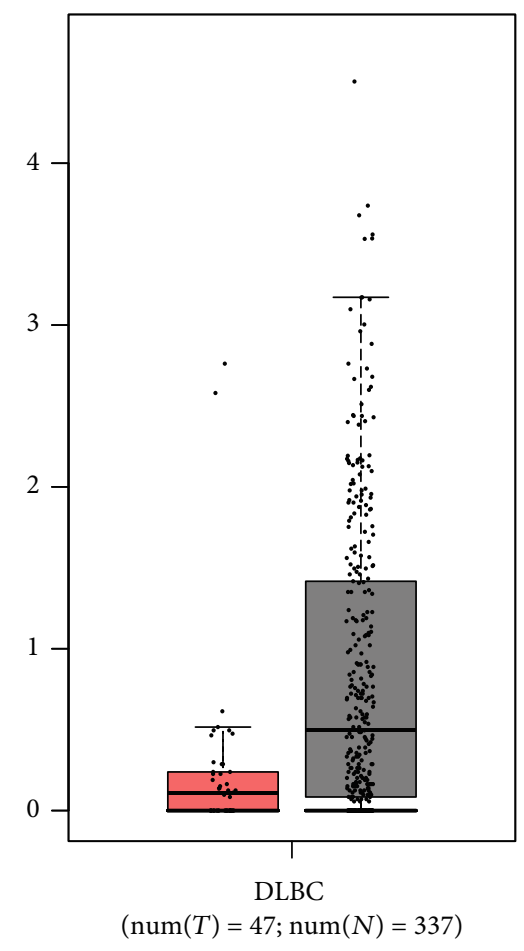

FIgure 7: Candidate gene risk assessment using GEPIA. Red represents high expression, and gray represents low expression. There are 47 patients with DLBCL and 337 normal patients.

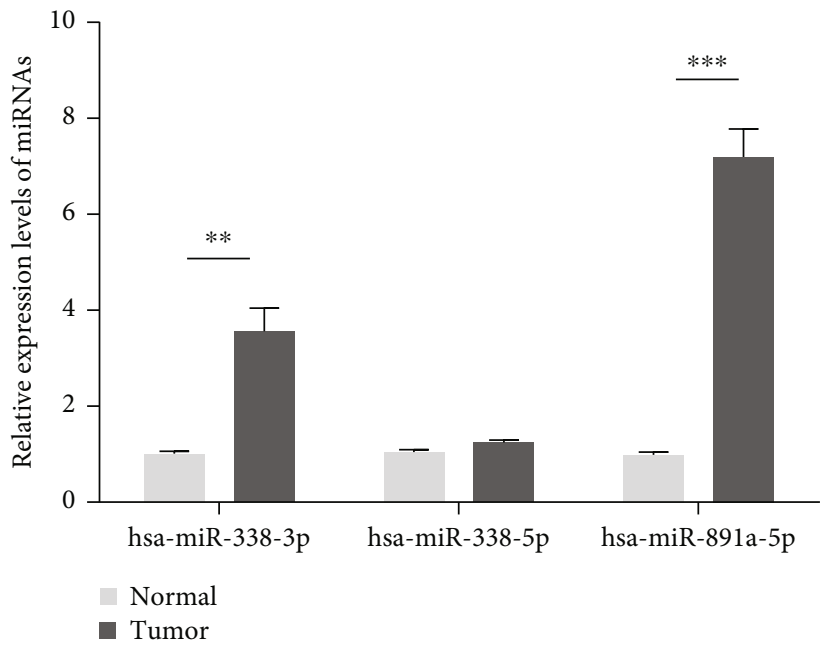

FIgURE 8: RT-qPCR showing the expression levels of hsa-miR-3383p, hsa-miR-338-5p, and hsa-miR-891a-5p in DLBCL. ${ }^{* *} p<0.01$; ${ }^{* * *} p<0.001$.

by WGCNA. And we found that the purple module was closely associated with histological type. Further analysis suggested that three candidate genes (MAFA-AS1, hsa-mir-338, and hsa-mir-891a) were significantly related with clinical outcomes. Therefore, our findings revealed a coexpression module related with histological type and identified three candidate genes (MAFA-AS1, hsa-mir-338, and hsa-mir- 

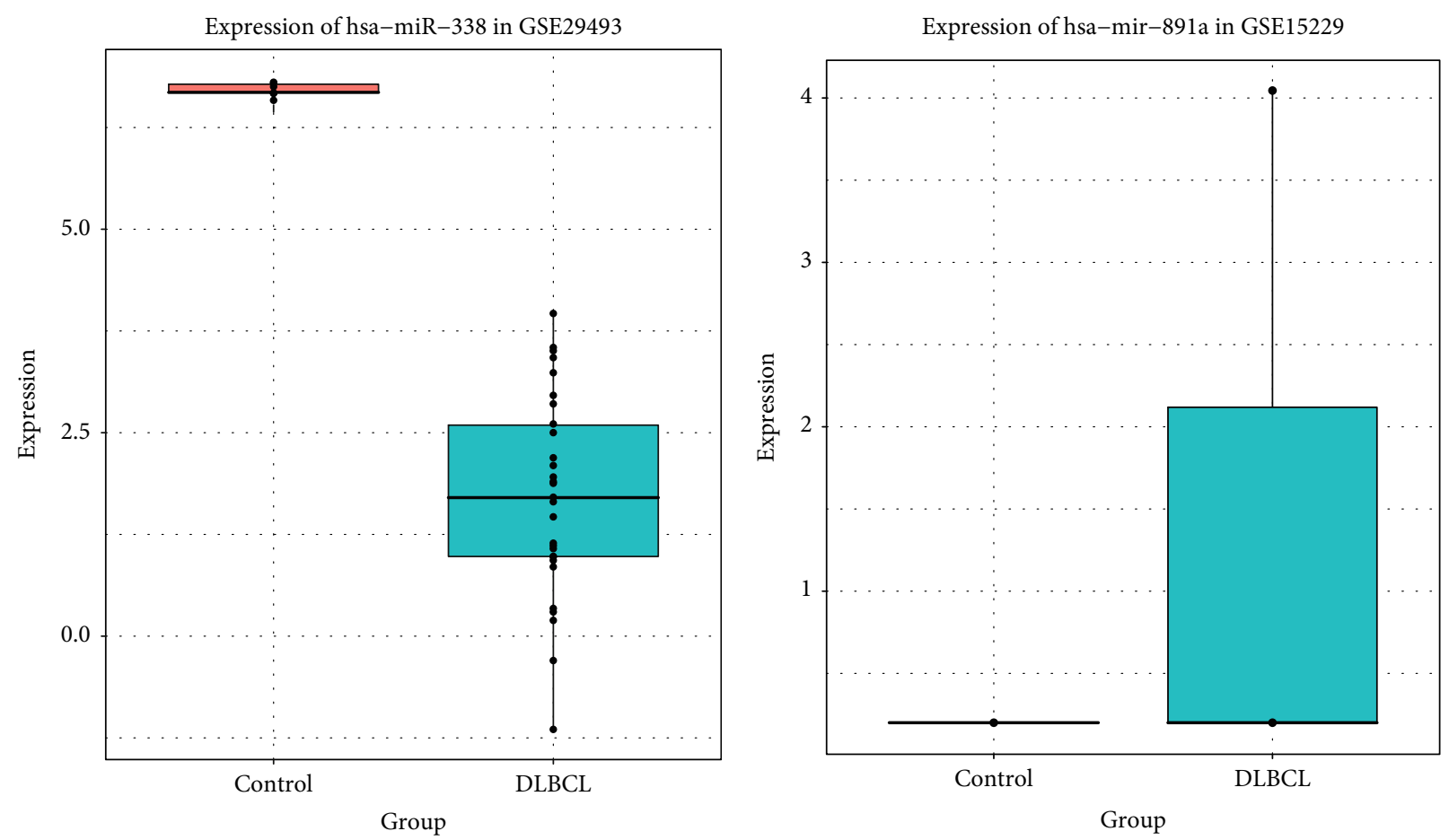

Group
白 Control
由 DLBCL

Group

白 Control

白 DLBCL

(a)

(b)

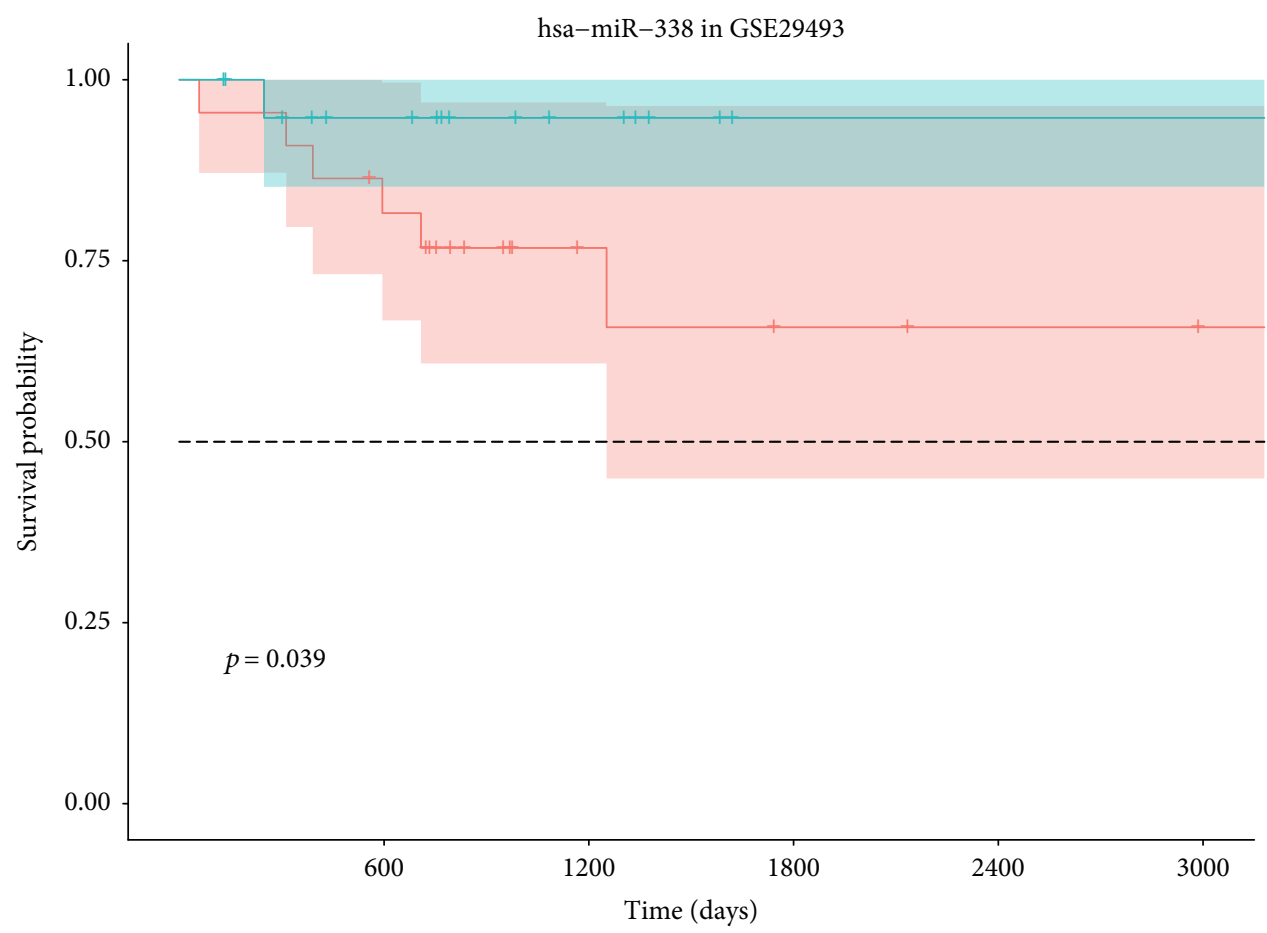

Strata

+ Group=high

+ Group=low

(c)

Figure 9: Continued. 


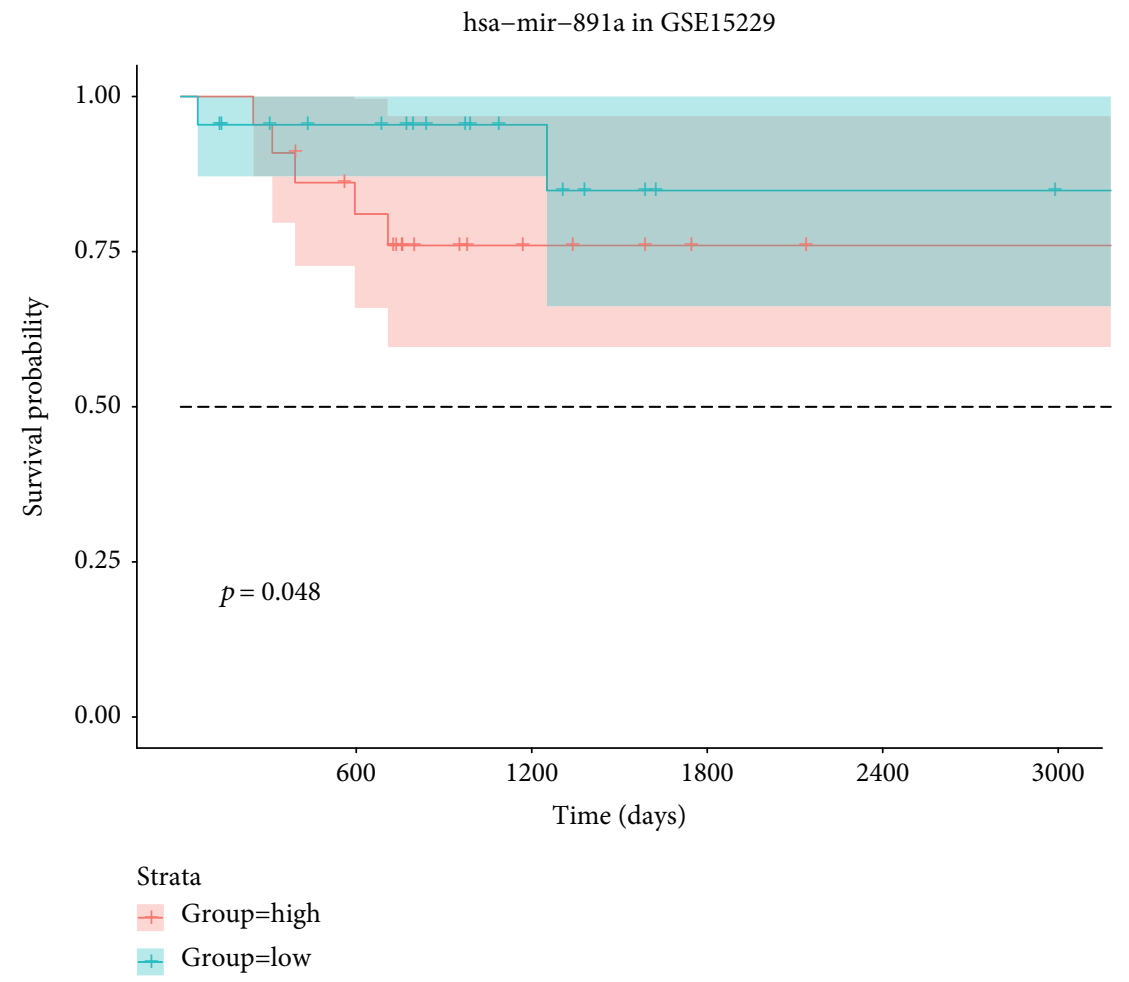

(d)

FIGURE 9: Validation of candidate prognostic miRNAs in independent datasets. The differences in expression patterns of hsa-miR-338 (a) and hsa-miR-891a (b) between DLBCL and controls. Overall survival analysis of hsa-miR-338 (c) and hsa-miR-891a (d) in DLBCL.

891a) that possessed potential value as prognostic biomarkers or potential therapeutic targets of DLBCL.

\section{Abbreviations}

DLBCL: Diffuse large B-cell lymphoma

IPI: $\quad$ Prognostic index

RNA-seq: RNA sequencing

TCGA: The Cancer Genome Atlas

WGCNA: Weighted gene coexpression network analysis

FPKM: Fragments per kilobase of transcript per million mapped reads

MAD: Median absolute deviation

ME: $\quad$ Module eigengene

GO: $\quad$ Gene Ontology

KEGG: $\quad$ Kyoto Encyclopedia of Genes and Genomes

GEPIA: Gene Expression Profiling Interactive Analysis

BP: $\quad$ Biological processes

CC: $\quad$ Cellular components

MF: $\quad$ Molecular functions.

\section{Data Availability}

The datasets analyzed during the current study are available from the corresponding author on reasonable request.

\section{Conflicts of Interest}

The authors declare no conflicts of interest.

\section{Authors' Contributions}

Jianjun Xiao and Xuemei Wang contributed equally to this work.

\section{References}

[1] C. Ruiduo, D. Ying, and W. Qiwei, "CXCL9 promotes the progression of diffuse large B-cell lymphoma through upregulating $\beta$-catenin," Biomedicine \& Pharmacotherapy, vol. 107, pp. 689-695, 2018.

[2] S. E. Arthur, A. Jiang, B. M. Grande et al., "Genome-wide discovery of somatic regulatory variants in diffuse large B-cell lymphoma," Nature Communications, vol. 9, no. 1, p. 4001, 2018.

[3] S. J. Schuster, M. R. Bishop, C. S. Tam et al., "Tisagenlecleucel in adult relapsed or refractory diffuse large B-cell lymphoma," The New England Journal of Medicine, vol. 380, no. 1, pp. 4556, 2019.

[4] M. Mian, L. Marcheselli, A. Rossi et al., "A diachroniccomparative analysis for the identification of the most powerful prognostic index for localized diffuse large B-cell lymphoma," Annals of Oncology, vol. 25, no. 12, pp. 23982404, 2014.

[5] J. Jelicic, T. S. Larsen, M. Maksimovic, and G. Trajkovic, "Available prognostic models for risk stratification of diffuse large B cell lymphoma patients: a systematic review," Critical Reviews in Oncology/Hematology, vol. 133, pp. 1-16, 2019.

[6] D. Rossi, F. Diop, E. Spaccarotella et al., "Diffuse large B-cell lymphoma genotyping on the liquid biopsy," Blood, vol. 129, no. 14, pp. 1947-1957, 2017. 
[7] J. R. Pon and M. A. Marra, "Clinical impact of molecular features in diffuse large B-cell lymphoma and follicular lymphoma," Blood, vol. 127, no. 2, pp. 181-186, 2016.

[8] Z. Wang, M. Gerstein, and M. Snyder, "RNA-Seq: a revolutionary tool for transcriptomics," Nature Reviews Genetics, vol. 10, no. 1, pp. 57-63, 2009.

[9] J. Lovén, D. A. Orlando, A. A. Sigova et al., "Revisiting global gene expression analysis," Cell, vol. 151, no. 3, pp. 476-482, 2012.

[10] J. A. Botía, The United Kingdom Brain Expression Consortium, J. Vandrovcova et al., "An additional k-means clustering step improves the biological features of WGCNA gene coexpression networks," BMC Systems Biology, vol. 11, no. 1, article 47, 2017.

[11] X. Lu, Y. Deng, L. Huang, B. Feng, and B. Liao, “A coexpression modules based gene selection for cancer recognition," Journal of Theoretical Biology, vol. 362, pp. 75-82, 2014.

[12] X. Liu, A. X. Hu, J. L. Zhao, and F. L. Chen, "Identification of key gene modules in human osteosarcoma by co-expression analysis weighted gene co-expression network analysis (WGCNA)," Journal of Cellular Biochemistry, vol. 118, no. 11, pp. 3953-3959, 2017.

[13] T. Sun, Y. Song, H. Yu, and X. Luo, "Identification of lncRNA TRPM2-AS/miR-140-3p/PYCR1 axis's proliferates and antiapoptotic effect on breast cancer using co-expression network analysis," Cancer Biology \& Therapy, pp. 1-14, 2019.

[14] Q. Wan, J. Tang, Y. Han, and D. Wang, "Co-expression modules construction by WGCNA and identify potential prognostic markers of uveal melanoma," Experimental Eye Research, vol. 166, pp. 13-20, 2018.

[15] L. Zhang, W. Kang, X. Lu, S. Ma, L. Dong, and B. Zou, "Weighted gene co-expression network analysis and connectivity map identifies lovastatin as a treatment option of gastric cancer by inhibiting HDAC2," Gene, vol. 681, pp. 15-25, 2019.

[16] X. G. Zhou, X. L. Huang, S. Y. Liang et al., "Identifying miRNA and gene modules of colon cancer associated with pathological stage by weighted gene co-expression network analysis," OncoTargets and Therapy, vol. 11, pp. 2815-2830, 2018.

[17] P. Langfelder and S. Horvath, "WGCNA: an R package for weighted correlation network analysis," BMC Bioinformatics, vol. 9, no. 1, p. 559, 2008.

[18] G. Yu, L. G. Wang, Y. Han, and Q. Y. He, "clusterProfiler: an R package for comparing biological themes among gene clusters," OMICS, vol. 16, no. 5, pp. 284-287, 2012.

[19] Z. Tang, C. Li, B. Kang, G. Gao, C. Li, and Z. Zhang, "GEPIA: a web server for cancer and normal gene expression profiling and interactive analyses," Nucleic Acids Research, vol. 45, no. W1, pp. W98-W102, 2017.

[20] J. H. Pan, H. Zhou, L. Cooper et al., "LAYN is a prognostic biomarker and correlated with immune infiltrates in gastric and colon cancers," Frontiers in Immunology, vol. 10, p. 6, 2019.

[21] S. K. Leivonen, K. Icay, K. Jäntti et al., "MicroRNAs regulate key cell survival pathways and mediate chemosensitivity during progression of diffuse large B-cell lymphoma," Blood Cancer Journal, vol. 7, no. 12, p. 654, 2017.

[22] S. Mizuno, I. Hanamura, A. Ota et al., "Establishment and characterization of a novel vincristine-resistant diffuse large B-cell lymphoma cell line containing the 8q24 homogeneously staining region," FEBS Open Bio, vol. 8, no. 12, pp. 1977-1991, 2018 .
[23] J. W. Liang, Z. Y. Fang, Y. Huang et al., "Application of weighted gene co-expression network analysis to explore the key genes in Alzheimer's disease," Journal of Alzheimer's Disease, vol. 65, no. 4, pp. 1353-1364, 2018.

[24] B. Rahmani, M. T. Zimmermann, D. E. Grill et al., "Recursive indirect-paths modularity (RIP-M) for detecting community structure in RNA-Seq co-expression networks," Frontiers in Genetics, vol. 7, p. 80, 2016.

[25] L. Yin, Z. Cai, B. Zhu, and C. Xu, "Identification of key pathways and genes in the dynamic progression of HCC based on WGCNA," Genes, vol. 9, no. 2, p. 92, 2018.

[26] M. Giulietti, G. Occhipinti, G. Principato, and F. Piva, "Identification of candidate miRNA biomarkers for pancreatic ductal adenocarcinoma by weighted gene co-expression network analysis," Cellular Oncology, vol. 40, no. 2, pp. 181-192, 2017.

[27] M. Sakurai, D. Karigane, H. Kasahara et al., "Geriatric screening tools predict survival outcomes in older patients with diffuse large B cell lymphoma," Annals of Hematology, vol. 98, no. 3, pp. 669-678, 2019.

[28] W. Klapper, M. Kreuz, C. W. Kohler et al., "Patient age at diagnosis is associated with the molecular characteristics of diffuse large B-cell lymphoma," Blood, vol. 119, no. 8, pp. 1882-1887, 2012.

[29] S. Bhalla, A. M. Evens, B. Dai, S. Prachand, L. I. Gordon, and R. B. Gartenhaus, "The novel anti-MEK small molecule AZD6244 induces BIM-dependent and AKT-independent apoptosis in diffuse large B-cell lymphoma," Blood, vol. 118, no. 4, pp. 1052-1061, 2011.

[30] W. Cui, Y. Cai, W. Wang et al., "Frequent copy number variations of PI3K/AKT pathway and aberrant protein expressions of PI3K subunits are associated with inferior survival in diffuse large B cell lymphoma," Journal of Translational Medicine, vol. 12, no. 1, p. 10, 2014.

[31] A. Majchrzak, M. Witkowska, and P. Smolewski, "Inhibition of the PI3K/Akt/mTOR signaling pathway in diffuse large B-cell lymphoma: current knowledge and clinical significance," Molecules, vol. 19, no. 9, pp. 14304-14315, 2014.

[32] N. Beauchemin and A. Arabzadeh, "Carcinoembryonic antigen-related cell adhesion molecules (CEACAMs) in cancer progression and metastasis," Cancer Metastasis Reviews, vol. 32, no. 3-4, pp. 643-671, 2013.

[33] M. Xin, X. W. Dong, and X. L. Guo, "Role of the interaction between galectin-3 and cell adhesion molecules in cancer metastasis," Biomedicine \& Pharmacotherapy, vol. 69, pp. 179-185, 2015.

[34] N. Wang, Q. Wang, J. Chi et al., "Carcinoembryonic antigen cell adhesion molecule 1 inhibits the antitumor effect of neutrophils in tongue squamous cell carcinoma," Cancer Science, vol. 110, no. 2, pp. 519-529, 2018.

[35] Y. Feng, M. Zhong, S. Zeng et al., "Exosome-derived miRNAs as predictive biomarkers for diffuse large B-cell lymphoma chemotherapy resistance," Epigenomics, vol. 11, no. 1, pp. 35-51, 2019.

[36] B. He, F. Yan, and C. Wu, “Overexpressed miR-195 attenuated immune escape of diffuse large B-cell lymphoma by targeting PD-L1," Biomedicine \& Pharmacotherapy, vol. 98, pp. 95101, 2018.

[37] F. Q. Zhu, L. Zeng, N. Tang et al., "MicroRNA-155 downregulation promotes cell cycle arrest and apoptosis in diffuse large B-cell lymphoma," Oncology Research, vol. 24, no. 6, pp. 415427, 2016. 
[38] Q. Fan, X. Meng, H. Liang et al., "miR-10a inhibits cell proliferation and promotes cell apoptosis by targeting BCL6 in diffuse large B-cell lymphoma," Protein \& Cell, vol. 7, no. 12, pp. 899-912, 2016.

[39] N. Sun, C. Y. Wang, Y. Q. Sun et al., “Down-regulated miR$148 \mathrm{~b}$ increases resistance to CHOP in diffuse large B-cell lymphoma cells by rescuing Ezrin," Biomedicine \& Pharmacotherapy, vol. 106, pp. 267-274, 2018.

[40] Q. Xue, K. Sun, and H. J. Deng, "MicroRNA-338-3p inhibits colorectal carcinoma cell invasion and migration by targeting smoothened," Japanese Journal of Clinical Oncology, vol. 44, no. 1, pp. 13-21, 2014.

[41] M. Yang, R. Liu, J. Sheng et al., "Differential expression profiles of microRNAs as potential biomarkers for the early diagnosis of esophageal squamous cell carcinoma," Oncology Reports, vol. 29, no. 1, pp. 169-176, 2012.

[42] Z. Y. Yan, Z. Q. Luo, L. J. Zhang, J. Li, and J. Q. Liu, "Integrated analysis and microRNA expression profiling identified seven miRNAs associated with progression of oral squamous cell carcinoma," Journal of Cellular Physiology, vol. 232, no. 8, pp. 2178-2185, 2017. 